Copyright ( 2013 IEEE. Personal use of this material is permitted. Permission from IEEE must be obtained for all other uses, in any current or future media, including reprinting/republishing this material for advertising or promotional purposes, creating new collective works, for resale or redistribution to servers or lists, or reuse of any copyrighted component of this work in other works. 


\title{
Design of Steerable Spherical Broadband Beamformers with Flexible Sensor Configurations
}

\author{
*Chiong Ching Lai, Sven Nordholm, Yee Hong Leung
}

\begin{abstract}
In broadband beamformer applications with dynamically moving sources, it can be important to have a simple mechanism to steer the main-beam. It can also be desirable if the beampattern of the beamformer is invariant to the look direction. A number of design methods for such beamformers, based on the spherical harmonic transform, have been reported in the literature. However, these methods require the sensor positions to satisfy a certain condition which may conflict with practical considerations. This paper proposes a design method which obviates this restriction thus allowing for spherical arrays with arbitrary sensor configurations. Moreover, for a comparable level of performance and computational complexity to the existing spherical harmonic beamformers, the proposed beamformer requires fewer sensors. The trade-off is that the design of the beamformer now depends on the sensor positions. Other considerations, such as the effects of array mis-orientation and robustness, are also discussed in this paper and illustrated by design examples.
\end{abstract}

Index Terms - spherical harmonics, broadband, orthonormality, steerable, sensor configuration

\section{INTRODUCTION}

Compared to azimuth only sensor array beamforming, azimuth-elevation sensor array beamforming offers an additional spatial dimension for acoustic reception, which is useful in applications that requires azimuth-elevation spatial sound reception such as audio surveillance, teleconferencing and source localisation [1]. For these applications, spherical arrays can be attractive because their symmetrical structure allows for beampatterns that are invariant to the look direction. There exist a number of methods to design spherical array beamformers but a particularly appealing approach is through the spherical harmonic transform framework since the transform is closely matched to the spherical array [2], [3]. The key benefits of this design approach are: a) straightforward beam steering with steering invariant beampatterns, $b$ ) independence of the sensor sampling scheme, as long as the sensor positions satisfy an orthonormality criterion, and c) usually less computationally intensive than filter-and-sum beamformers.

Initial studies on this subject were carried out by Meyer and Elko [2], and Abhayapala and Ward [3], who proposed beamformer designs (commonly known as spherical beamformers) based on the spherical harmonic decomposition of the received sound field. Their designs allow for simple beam steering by

The authors are with Dept. of Electrical and Computer Engineering, Curtin University, GPO Box U1987 Perth, Western Australia 6845. Tel: (+618) 9266 7039; (+618) 9266 7439; (+618) 9266 7393. Email: chiong.lai@ curtin.edu.au; s.nordholm@curtin.edu.au; y.leung@curtin.edu.au. This research is supported in part by Sensear Pty Ltd and Linkage Grant LP100100433 by the Australian Research Council (ARC).

EDICS: AUD-LMAP means of modulation, similar to the steering mechanism in [4]. Since then, studies in this area have grown which include designs of optimal beamformers, adaptive beamformers and nearfield beamformers in the spherical harmonic domain [5], [6], [7].

As mentioned, an issue with these spherical beamformer designs is that most of them require the sensor configuration to satisfy an orthonormality criterion in order to avoid spatial aliasing from high order spherical harmonics [8]. Accordingly, different sensor sampling schemes such as equiangle sampling [9], Gaussian sampling [10], [11] and uniform or quasiuniform sampling [12], [13] have been proposed to satisfy the orthonormality criterion for spherical harmonics up to a certain order $N$. However, these sampling schemes require more than $1.5(N+1)^{2}$ sensors in practice [14]. Moreover, the sensor configurations given by these sampling schemes are very stringent and may not be feasible in some practical applications where the array is to be mounted.

Li and Duraiswami [15] studied the impact of orthonormality errors in the sensor configuration on the array performance. They proposed a spherical beamformer weight design method that explicitly satisfies the orthonormality property. Hence, their method introduces flexibility in the sensor configuration as the beamformer weights are used to satisfy the orthonormality criterion. However, in their design, beam steering is less straightforward and requires the beamformer weights to be changed for different look directions. The number of possible look directions are thus limited by the size of the look-up table.

In this paper, a method to combine the simple steering capability of spherical harmonic beamformers with the generality of the filter-and-sum beamfomers is proposed. This is achieved by including into the filter-and-sum beamformer structure, the modal decomposition and beam steering block from [2], [6]. The resulting structure is similar to the existing spherical harmonic beamformer structure, except that the filtering block is moved to the sensor end. Although this modification causes the independent sensor sampling and computational advantages of the existing spherical harmonic beamformers to be lost, it allows for arbitrary sensor positions, and retains the simple steering and the steering-invariant beampattern properties of the existing spherical harmonic beamformers. This contrasts with the steerable broadband beamformers designed in the element space domain [16], [17], [18], and the filter-andsum beamformers with presteering [19], whose beampattern changes with the steering direction. In addition, the dimension of the design problem increases significantly when using methods such as [16], [18], which makes the scaling of the 
problem very difficult, and complicates their implementation.

This paper is organized as follows. Section II provides a brief discussion on the existing spherical beamformer design methods as well as the sensor sampling schemes. The proposed design formulation, in both frequency and time domains, are discussed in Section III and their properties are investigated in Section IV. Design examples are presented in Section V and the conclusions are drawn in Section VI.

\section{BACKGROUND}

\section{A. Spherical harmonics beamforming}

Consider a unit magnitude plane wave (farfield source model) impinging on a sphere with radius $a$ from direction $\Omega=(\theta, \phi)$ as shown in Fig. 2, where $\theta$ is the elevation angle and $\phi$ is the azimuth angle. The frequency domain expression of the total sound pressure as observed at a point $\tilde{\Omega}=(\tilde{\theta}, \tilde{\phi})$ on the sphere surface can be written as [3], [20]

$$
p(k a, \Omega, \tilde{\Omega})=\sum_{n=0}^{\infty} \sum_{m=-n}^{n} p_{n}^{m}(k a, \Omega) Y_{n}^{m}(\tilde{\Omega})
$$

where $k=2 \pi f / c$ is the wavenumber, $f$ is the frequency and $c$ is the speed of the propagating wave. $Y_{n}^{m}(\Omega)$ denotes the spherical harmonic of order $n$ and degree $m$ and is given by [20]

$$
Y_{n}^{m}(\Omega)=\sqrt{\frac{(2 n+1)}{4 \pi} \frac{(n-m) !}{(n+m) !}} P_{n}^{m}(\cos (\theta)) e^{i m \phi}
$$

where $P_{n}^{m}(\cos (\theta))$ is the associated Legendre polynomial of order $n$ and degree $m$, and $i=\sqrt{-1}$. The term $p_{n}^{m}(k a, \Omega)$, which is the spherical Fourier transform or spherical harmonic coefficient of $p(k a, \Omega, \tilde{\Omega})$, is given by

$$
p_{n}^{m}(k a, \Omega)=\int_{\tilde{\Omega} \in S^{2}} p(k a, \Omega, \tilde{\Omega})\left[Y_{n}^{m}(\tilde{\Omega})\right]^{*} d \tilde{\Omega}
$$

where the integration is evaluated over the whole surface of the unit sphere $S^{2}$ and the superscript $*$ denotes complex conjugate. Note that (3) is the spherical harmonic transform of $p(k a, \Omega, \tilde{\Omega})$ and (1) is its inverse [20]. The explicit expression for $p_{n}^{m}(k a, \Omega)$, which can be obtained by solving the wave equation, is given by

$$
p_{n}^{m}(k a, \Omega)=b_{n}(k a)\left[Y_{n}^{m}(\Omega)\right]^{*} .
$$

The coefficient $b_{n}(k a)$ depends on the type of sphere. For example, for an open sphere (sensors are "floating" in free space) and a rigid sphere (sensors mounted on surface of solid sphere) [20],

$b_{n}(k a)= \begin{cases}4 \pi i^{n} j_{n}(k a), & \text { open sphere } \\ 4 \pi i^{n}\left(j_{n}(k a)-\frac{j_{n}^{\prime}(k a)}{h_{n}^{\prime}(k a)} h_{n}(k a)\right), & \text { rigid sphere }\end{cases}$

where $j_{n}(\cdot)$ and $h_{n}(\cdot)$ are respectively the $n^{\text {th }}$ order spherical Bessel and Hankel function of the first kind, and $j_{n}^{\prime}(\cdot)$ and $h_{n}^{\prime}(\cdot)$ are their derivatives with respect to their arguments.

The basic principle of spherical harmonic beamforming is to use the spherical harmonic framework to decompose

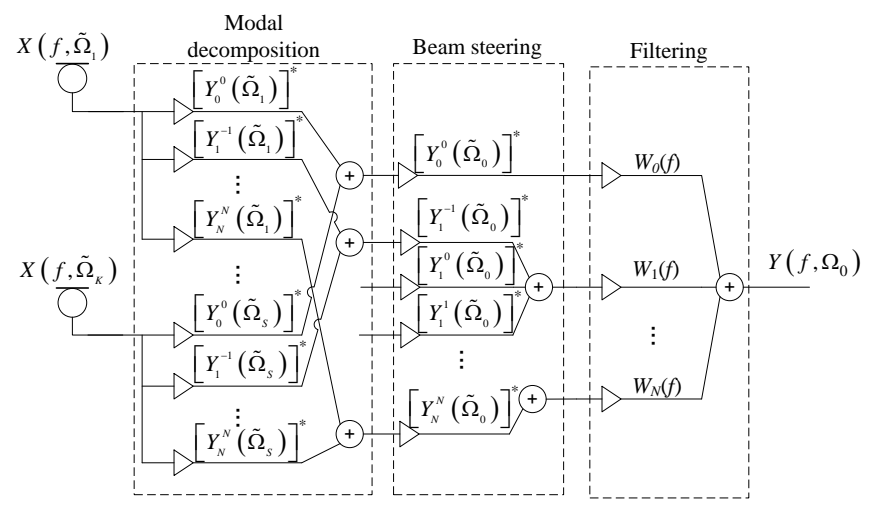

Figure 1. Existing spherical harmonic beamformer structure.

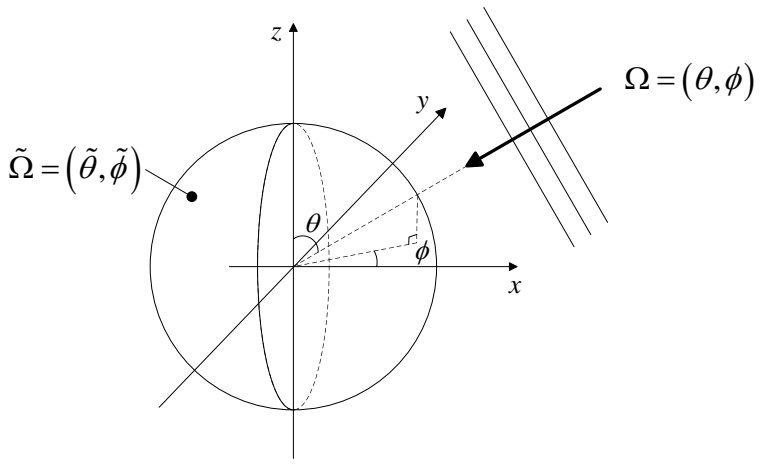

Figure 2. Plane wave incident on spherical array.

the sound field received by a spherical array into orthogonal components. These components are then linearly combined to achieve the desired beampattern [15], [20]. The resulting beamformer structure is shown in Fig. 1. In the first stage, the received signals are decomposed by the modal decomposition block into their spherical harmonic components. The spherical harmonic components components are then modulated by the steering block to favour reception from the desired look direction. Finally, frequency response and beampattern shaping are performed by the filtering block.

A limitation of the existing spherical beamformer design methods such as [2], [6], [21], in that the sensor positions are restricted by the orthonormality criterion

$$
\sum_{s} \alpha_{s}\left[Y_{n}^{m}\left(\tilde{\Omega}_{s}\right)\right]^{*} Y_{n^{\prime}}^{m^{\prime}}\left(\tilde{\Omega}_{s}\right)=\delta_{m-m^{\prime}} \delta_{n-n^{\prime}}
$$

where $\tilde{\Omega}_{s}=\left(\tilde{\theta}_{s}, \tilde{\phi}_{s}\right)$ is the position and $\alpha_{s} \in \mathbb{R}$ is the scaling factor of the $s^{\text {th }}$ sensor.

\section{B. Spatial sampling on a sphere}

In order to satisfy the sensor orthonormality criterion (6), different sampling schemes have been reported. The first sampling scheme is equiangle sampling [9], where at least $4(N+1)^{2}$ sensors are required to resolve spherical harmonics up to the $N^{t h}$ order without spatial aliasing. As the name implies, this scheme requires a constant angle difference between adjacent sensors, which can be advantageous if azimuthal and elevation symmetry of the array is required. However, it 
requires a large number of sensors as compared to the other sampling schemes.

The second sampling scheme is Gaussian quadrature sampling [10], [11]. It requires at least $2(N+1)^{2}$ sensors. Under this scheme, the azimuth angle remains uniformly spaced but the elevation angle is no longer equally spaced. Although this sampling scheme requires less sensors than the equiangle sampling scheme, the non-uniformity of the sensor spacing in elevation may be undesirable in some applications.

The third sampling scheme is uniform or quasi-uniform sampling [12], [13], where the distances (as measured along the arc of the sphere surface) between adjacent sensors are constant. This sampling scheme limits the sensor configuration to a specific set of geometries known as platonic solids. Hardin and Sloane [14] show that the number of sensors required for this sampling scheme is at least $(N+1)^{2}$, but in practice, more than $1.5(N+1)^{2}$ are required. It should be noted that in contrast to equiangle and Gaussian quadrature sampling, the criterion (6) under this sampling scheme may not be completely satisfied.

The main limitation of all the above sampling schemes is that the sensor configuration is fixed. This may not be feasible in cases where regularity in the spherical array cannot be achieved.

\section{PROPOSED SPHERICAL BEAMFORMER DESIGN}

\section{A. Frequency domain design}

Consider again a sphere with radius $a$. Suppose its surface consists of a continuous receiver (continuous sensor array), and let a point on the surface be denoted as $\tilde{\Omega}=(\tilde{\theta}, \tilde{\phi})$. Suppose the output of the continuous array is weighted with a frequency and location (on the surface of the sphere) dependent complex weight $w(f, \tilde{\Omega})$. The beampattern, which is defined as the beamformer response to a unit magnitude wave arriving from direction $\Omega$, is then given by

$$
A(f, \Omega)=\oint_{\tilde{\Omega} \in S^{2}} p(k a, \Omega, \tilde{\Omega}) w^{*}(f, \tilde{\Omega}) d \tilde{\Omega} .
$$

Substituting (1) and (4) into (7), and restricting the summation to the $N^{t h}$ order spherical harmonic, results in

$$
\begin{aligned}
A(f, \Omega)= & \sum_{n=0}^{N} \sum_{m=-n}^{n} b_{n}(k a)\left[Y_{n}^{m}(\Omega)\right]^{*} \\
& \times \oint_{\tilde{\Omega} \in S^{2}} w^{*}(f, \tilde{\Omega}) Y_{n}^{m}(\tilde{\Omega}) d \tilde{\Omega} .
\end{aligned}
$$

In order to incorporate an ability to rotate the beampattern to any direction $\Omega_{0}=\left(\theta_{0}, \phi_{0}\right)$, the term $Y_{n}^{m}\left(\Omega_{0}\right)$ is included in the right hand side of (8) [2], [6], which yields

$$
\begin{aligned}
B\left(f, \Omega, \Omega_{0}\right) & =\sum_{n=0}^{N} \sum_{m=-n}^{n}\left[Y_{n}^{m}(\Omega)\right]^{*} Y_{n}^{m}\left(\Omega_{0}\right) \\
& \times\left(b_{n}(k a) \oint_{\tilde{\Omega} \in S^{2}} w^{*}(f, \tilde{\Omega}) Y_{n}^{m}(\tilde{\Omega}) d \tilde{\Omega}\right) .
\end{aligned}
$$

Let the desired beampattern with the main-beam pointing at $\Omega_{0}$ be given by

$$
B_{d}\left(f, \Omega, \Omega_{0}\right)=\sum_{n=0}^{N} \sum_{m=-n}^{n} \alpha_{n}^{m}(f)\left[Y_{n}^{m}(\Omega)\right]^{*} Y_{n}^{m}\left(\Omega_{0}\right) .
$$

Equation (10) is a linear combination of the complex conjugate of all spherical harmonics up to order $N$ where the beam shaping complex weights $\alpha_{n}^{m}(f)$ can be determined from [2], [5]. Equating the expression for the actual beampattern in (9) with the desired beampattern in (10) yields

$$
b_{n}(k a) \oint_{\tilde{\Omega} \in S^{2}} w^{*}(f, \tilde{\Omega}) Y_{n}^{m}(\tilde{\Omega}) d \tilde{\Omega}=\alpha_{n}^{m}(f)
$$

for all $n$ and $m$. But

$$
[S\{w(f, \tilde{\Omega})\}]^{*}=\oint_{\tilde{\Omega} \in S^{2}} w^{*}(f, \tilde{\Omega}) Y_{n}^{m}(\tilde{\Omega}) d \tilde{\Omega},
$$

where $S\{\cdot\}$ is the spherical harmonic transform. The solution for $w(f, \tilde{\Omega})$ is thus given by

$$
\begin{aligned}
w(f, \tilde{\Omega}) & =S^{-1}\left\{\left[\frac{\alpha_{n}^{m}(f)}{b_{n}(k a)}\right]^{*}\right\} \\
& =\sum_{n=0}^{\infty} \sum_{m=-n}^{n}\left[\frac{\alpha_{n}^{m}(f)}{b_{n}(k a)}\right]^{*} Y_{n}^{m}(\tilde{\Omega}) .
\end{aligned}
$$

Although (13) gives the analytical expression for the continuous spherical beamformer weights, it is not practical due to the infinite summation.

To make the design practical, the continuous sensor array on the sphere surface is discretised into $K$ discrete points or sensors, indexed by $s=1, \ldots, K$. Equation (9) then becomes

$$
\begin{aligned}
B\left(f, \Omega, \Omega_{0}\right) & \approx \sum_{n=0}^{N} \sum_{m=-n}^{n}\left[Y_{n}^{m}(\Omega)\right]^{*} Y_{n}^{m}\left(\Omega_{0}\right) \\
& \times\left(b_{n}(k a) \sum_{s=1}^{K} w^{*}\left(f, \tilde{\Omega}_{s}\right) Y_{n}^{m}\left(\tilde{\Omega}_{s}\right)\right)
\end{aligned}
$$

where the scaling factor $\alpha_{s}$ for the $s^{t h}$ sensor has been merged into $w^{*}\left(f, \tilde{\Omega}_{s}\right)$. The equation to find the beamformer weights (by equating (14) with (10)) is then given by

$$
b_{n}(k a) \sum_{s=1}^{K} w^{*}\left(f, \tilde{\Omega}_{s}\right) Y_{n}^{m}\left(\tilde{\Omega}_{s}\right)=\alpha_{n}^{m}(f)
$$

for $n \in\{0,1, \ldots, N\}, m \in\{-n,-n+1, \ldots, n\}$ and $f \in \Omega_{p b}$ where $\Omega_{p b}$ is the spectral range of interest. Let

$$
\begin{aligned}
\mathbf{Y} & =\left[\left(\mathbf{y}_{0}^{0}\right),\left(\mathbf{y}_{1}^{-1}\right),\left(\mathbf{y}_{1}^{0}\right),\left(\mathbf{y}_{1}^{1}\right), \ldots,\left(\mathbf{y}_{N}^{N}\right)\right]^{\mathrm{T}} \\
\mathbf{y}_{n}^{m} & =\left[Y_{n}^{m}\left(\tilde{\Omega}_{1}\right), \ldots, Y_{n}^{m}\left(\tilde{\Omega}_{K}\right)\right]^{\mathrm{T}} \\
\mathbf{w}(f) & =\left[w^{*}\left(f, \tilde{\Omega}_{1}\right), \ldots, w^{*}\left(f, \tilde{\Omega}_{K}\right)\right]^{\mathrm{T}} \\
\boldsymbol{\alpha}(f) & =\left[\alpha_{0}^{0}(f), \alpha_{1}^{-1}(f), \alpha_{1}^{0}(f), \alpha_{1}^{1}(f), \ldots, \alpha_{N}^{N}(f)\right]^{\mathrm{T}} \\
\mathbf{B}(k a) & =\operatorname{diag}\left\{b_{0}(k a) \otimes \mathbf{1}_{1}, \ldots, b_{N}(k a) \otimes \mathbf{1}_{(2 N+1)}\right\}
\end{aligned}
$$




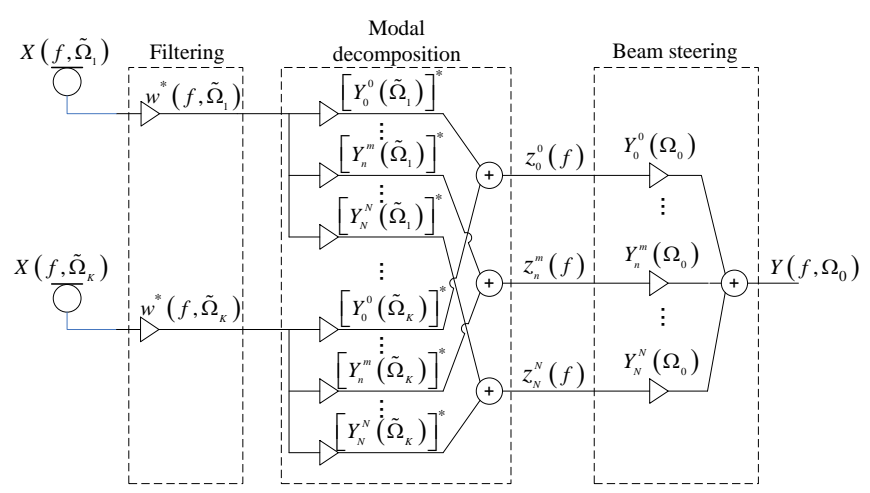

Figure 3. Proposed frequency domain beamformer structure.

where $\mathbf{1}_{J}$ is a length $J$ row vector with all unity elements, and $\otimes$ denotes Kronecker product. Using (16) - (20), equation (15) can be rewritten in matrix form as

$$
\mathbf{B}(k a) \mathbf{Y} \mathbf{w}(f)=\boldsymbol{\alpha}(f)
$$

which has analytical solution (in the least square sense)

$$
\mathbf{w}(f)=\left(\mathbf{Y}^{\mathrm{H}} \mathbf{B}^{\mathrm{H}}(k a) \mathbf{B}(k a) \mathbf{Y}\right)^{-1} \mathbf{Y}^{\mathrm{H}} \mathbf{B}^{\mathrm{H}}(k a) \boldsymbol{\alpha}(f)
$$

If $\mathbf{B}(k a) \mathbf{Y}$ is invertible, then (22) reduces to

$$
\mathbf{w}(f)=(\mathbf{B}(k a) \mathbf{Y})^{-1} \boldsymbol{\alpha}(f) .
$$

The Fourier transform of the output signal for the proposed beamformer is given by

$$
Y\left(f, \Omega_{0}\right)=\sum_{n=0}^{N} \sum_{m=-n}^{n} z_{n}^{m}(f) Y_{n}^{m}\left(\Omega_{0}\right)
$$

where the term $Y_{n}^{m}\left(\Omega_{0}\right)$ steers the main-beam to direction $\Omega_{0}$, and the modal decomposition of the intermediate output $z_{n}^{m}(f)$ is given by

$$
z_{n}^{m}(f)=\sum_{s=1}^{K}\left[X\left(f, \tilde{\Omega}_{s}\right) w^{*}\left(f, \tilde{\Omega}_{s}\right)\right]\left[Y_{n}^{m}\left(\tilde{\Omega}_{s}\right)\right]^{*}
$$

with $X\left(f, \tilde{\Omega}_{s}\right)$ the Fourier transform of the received signal at the $s^{\text {th }}$ sensor. The structure of the proposed spherical beamformer is shown in Fig. 3. Compared to the existing spherical beamformer structure shown in Fig. 1, the filtering block has been moved to the sensor end in the proposed design. In the proposed structure, the filtering block performs an additional task of compensating for the arbitrary sensor positions, apart from frequency response and beampattern shaping. The modal decomposition and beam steering blocks allow simple beam steering with invariant beampattern by decomposing the outputs of the filtering block into their orthogonal modes and modulating those modes to the desired look direction.

It is known that the performance of beamformers will degrade in the presence of errors and their robustness can be measured in terms of white noise gain (WNG) [22], [5]. In the presence of spatially uncorrelated white noise with power spectral density $\sigma_{n}^{2}$, the noise-only output power of the proposed beamformer is given by

$$
P_{n}(f)=\sigma_{n}^{2} \sum_{s=1}^{K}\left|w\left(f, \tilde{\Omega}_{s}\right)\right|^{2} .
$$

The WNG is then given by

$$
W N G(f)=\frac{\sigma_{n}^{2}}{P_{n}(f)}=\frac{1}{\mathbf{w}^{\mathrm{H}}(f) \mathbf{w}(f)} .
$$

A constraint based on (27) can be included into the design (by minimising the 2-norm distance squared between $\mathbf{B}(k a) \mathbf{Y} \mathbf{w}(f)$ and $\boldsymbol{\alpha}(f))$ to improve robustness as follows

$$
\begin{array}{rc}
\min _{\mathbf{w}(f)} & \|\mathbf{B}(k a) \mathbf{Y} \mathbf{w}(f)-\boldsymbol{\alpha}(f)\|_{2}^{2} \\
\text { subject to } & W N G^{-1}(f) \leq \rho(f)
\end{array}
$$

where $\rho(\cdot)$ is a design parameter. The design problem (28) can be solved using optimisation toolboxes such as SeDuMi [23].

\section{B. Time domain design}

The frequency domain design in Section III-A can be transformed into the time domain. For the ideal case, i.e. continuous sensor array with continuous-time non-causal filters, the impulse response of the proposed beamformer design is given by (see (13))

$$
h(t, \tilde{\Omega})=F^{-1}\left\{S^{-1}\left\{\left[\frac{\alpha_{n}^{m}(f)}{b_{n}(k a)}\right]^{*}\right\}\right\}
$$

where $F\{\cdot\}$ denotes Fourier transform.

In the case of discrete-time implementation, the impulse response (29) is sampled at sampling frequency $f_{S}$ and truncated to finite length. These operations are equivalent to approximating each of the frequency dependent complex weight in (15) with a real $L$-tap FIR filter, i.e.

$$
w^{*}\left(f, \tilde{\Omega}_{s}\right) \approx \mathbf{h}_{s}^{\mathrm{T}} \mathbf{e}(f), \quad|f|<\frac{f_{S}}{2}
$$

where

$$
\begin{aligned}
\mathbf{h}_{s} & =\left[h_{s}(0), h_{s}(l), \ldots, h_{s}(L-1)\right]^{\mathrm{T}} \\
\mathbf{e}(f) & =\left[1, \exp \left(-i \frac{2 \pi f}{f_{S}}\right), \ldots, \exp \left(-i \frac{2 \pi f(L-1)}{f_{S}}\right)\right]^{\mathrm{T}} .
\end{aligned}
$$

In order to compensate for the inherent group delay in FIR filters, a negative predelay, typically chosen as [6]

$$
\eta(f)=\exp \left(i \frac{\pi f(L-1)}{f_{S}}\right)
$$

is also added to (15) to yield the beampattern expression

$$
\begin{aligned}
\mathcal{B}\left(f, \Omega, \Omega_{0}\right) & =\sum_{n=0}^{N} \sum_{m=-n}^{n}\left[Y_{n}^{m}(\Omega)\right]^{*} Y_{n}^{m}\left(\Omega_{0}\right) \\
& \times\left(b_{n}(k a) \sum_{s=1}^{K} \eta(f) \mathbf{h}_{s}^{\mathrm{T}} \mathbf{e}(f) Y_{n}^{m}\left(\tilde{\Omega}_{s}\right)\right)
\end{aligned}
$$


for the time domain design. The weights design expression is then given by

$$
b_{n}(k a) \sum_{s=1}^{K} \eta(f) \mathbf{h}_{s}^{\mathrm{T}} \mathbf{e}(f) Y_{n}^{m}\left(\tilde{\Omega}_{s}\right)=\alpha_{n}^{m}(f)
$$

for all $n, m$ and $f \in \Omega_{p b}$. Note that the term $\eta(f)$, which cannot be realized in practice, is only required to determine the beamformer weights during the design stage and is not required in the actual implementation of the beamformer. Let $\mathbf{h}=\left[\mathbf{h}_{1}^{\mathrm{T}}, \ldots, \mathbf{h}_{K}^{\mathrm{T}}\right]^{\mathrm{T}}$. Equation (35) can then be rewritten in matrix form as

$$
\left((\mathbf{B}(k a) \mathbf{Y}) \otimes\left(\eta(f) \mathbf{e}^{\mathrm{T}}(f)\right)\right) \mathbf{h}=\boldsymbol{\alpha}(f) \quad \forall f \in \Omega_{p b} .
$$

Suppose the frequency interval $\Omega_{p b}$ in (36) is discretised into $M>K L$ points and let

$$
\begin{gathered}
\mathbf{A}=\left[\left(\left(\mathbf{B}\left(k_{1} a\right) \mathbf{Y}\right) \otimes\left(\eta\left(f_{1}\right) \mathbf{e}^{\mathrm{T}}\left(f_{1}\right)\right)\right)^{\mathrm{T}}, \ldots,\right. \\
\left.\left(\left(\mathbf{B}\left(k_{M} a\right) \mathbf{Y}\right) \otimes\left(\eta\left(f_{M}\right) \mathbf{e}^{\mathrm{T}}\left(f_{M}\right)\right)\right)^{\mathrm{T}}\right]^{\mathrm{T}} \\
\boldsymbol{\alpha}=\left[\boldsymbol{\alpha}^{\mathrm{T}}\left(f_{1}\right), \boldsymbol{\alpha}^{\mathrm{T}}\left(f_{2}\right), \ldots, \boldsymbol{\alpha}^{\mathrm{T}}\left(f_{M}\right)\right]^{\mathrm{T}},
\end{gathered}
$$

(36) can be written compactly as

$$
\mathrm{Ah}=\boldsymbol{\alpha} .
$$

Solving (39) in the least squares sense, while constraining $\mathbf{h} \in$ $\mathbb{R}^{K L}$, yields

$$
\mathbf{h}=\operatorname{Re}\left\{\mathbf{A}^{\mathrm{H}} \mathbf{A}\right\}^{-1} \operatorname{Re}\left\{\mathbf{A}^{\mathrm{H}} \boldsymbol{\alpha}\right\}
$$

where $\operatorname{Re}\{\cdot\}$ denotes the real part.

The implementation structure of the proposed time domain beamformer can be derived following the same procedure in [6]. The time domain output signal of the beamformer is given by

$$
y(l)=\sum_{n=0}^{N} \sum_{s=1}^{K}\left(x_{s}(l) \star h_{s}(l)\right) \sum_{m=-n}^{n}\left[Y_{n}^{m}\left(\tilde{\Omega}_{s}\right)\right]^{*} Y_{n}^{m}\left(\Omega_{0}\right)
$$

where $\star$ denotes convolution. The summation of complex spherical harmonics for $m=-n, \ldots, n$ in (41) can be expanded into summation of only real terms as in

$$
\begin{aligned}
& \sum_{m=-n}^{n}\left[Y_{n}^{m}\left(\tilde{\Omega}_{s}\right)\right]^{*} Y_{n}^{m}\left(\Omega_{0}\right)=Y_{n}^{0}\left(\tilde{\Omega}_{s}\right) Y_{n}^{0}\left(\Omega_{0}\right) \\
& +2 \sum_{m=1}^{n}\left(\operatorname{Re}\left\{Y_{n}^{m}\left(\tilde{\Omega}_{s}\right)\right\} a_{n}^{m} P_{n}^{m}\left(\cos \left(\theta_{0}\right)\right) \cos \left(m \phi_{0}\right)\right. \\
& \left.\quad+\operatorname{Im}\left\{Y_{n}^{m}\left(\tilde{\Omega}_{s}\right)\right\}\right) a_{n}^{m} P_{n}^{m}\left(\cos \left(\theta_{0}\right)\right) \sin \left(m \phi_{0}\right)
\end{aligned}
$$

where

$$
a_{n}^{m}=\sqrt{\frac{(2 n+1)}{4 \pi} \frac{(n-m) !}{(n+m) !}} .
$$

Substituting (42) into (41) results in a real expression for the time domain output signal of the modal beamformer. The resulting implementation structure is shown in Fig. 4.
The noise-only beamformer output power (in the presense of spatially uncorrelated white noise with power spectral density $\left.\sigma_{n}^{2}\right)$ is [6]

$$
\begin{aligned}
\bar{P}_{n} & =\int_{\Omega} P_{n}(f) d f \\
& =\sum_{s=1}^{K} \int_{\Omega} \sigma_{n}^{2}\left|\mathbf{h}_{s}^{\mathrm{T}} \mathbf{e}(f)\right|^{2} d f \\
& =\sigma_{n}^{2} \mathbf{h}^{\mathrm{T}} \mathbf{h} .
\end{aligned}
$$

Its $\mathrm{WNG}$ is then given by

$$
W N G=\frac{\sigma_{n}^{2}}{\bar{P}_{n}}=\frac{1}{\mathbf{h}^{\mathrm{T}} \mathbf{h}} .
$$

Robustness in the design can be achieved by constraining (45) while minimising the 2-norm distance squared between $\mathbf{A h}$ and $\alpha$, resulting in

$$
\begin{array}{rc}
\min _{\mathbf{w}(f)} & \|\mathbf{A h}-\boldsymbol{\alpha}\|_{2}^{2} \\
\text { subject to } & W N G^{-1} \leq \rho .
\end{array}
$$

As with (28) the above design problem can be solved using optimisation toolboxes such as SeDuMi.

\section{PROPERTIES OF THE PROPOSED BEAMFORMER}

\section{A. Independence of orthonormality criterion}

The expressions for the proposed beamformer weights in (15) and (36) are derived without referencing the orthonormality criterion (6). This means that, unlike the existing spherical beamformer designs, the proposed beamformer is not restricted by the orthonormality criterion and hence, failure to satisfy it will not affect the performance of the proposed beamformer. In other words, in the proposed beamformer, the sensor placement can follow any arbitrary array configuration as long as the sensors are reasonably spread out. This provides freedom and flexibility in the sensor configuration, especially in cases where it is not possible to satisfy (6) or where an irregular spherical array is required.

\section{B. Reduction in number of sensors}

In the frequency domain design, the beamformer weights $w^{*}\left(f, \tilde{\Omega}_{s}\right)$ are required to satisfy (15) for all $n=0, \ldots N$ and $m=-n, \ldots, n$. Its matrix equivalent, given in (21), shows that it is indeed a linear system with $(N+1)^{2}$ equations. Hence, in order to satisfy all $(N+1)^{2}$ equations in (21), the length of the weight vector $\mathbf{w}(f)$ must be at least $(N+1)^{2}$. In other words, $K \geq(N+1)^{2}$ sensors are required to resolve spherical harmonics up to order $N$ without any spatial aliasing. Compared to the existing spherical beamformer designs which use the sampling schemes discussed in Section II-B, the proposed beamformer design requires fewer sensors. This is favourable in applications where the number of sensors is a limiting factor, such as for small, low cost spherical arrays.

As for the time domain design, which is merely an approximation of the frequency domain counterpart, it inherits the same properties. This is because they both have the same structure except for the filtering block (see Figs. 3 and 4), 


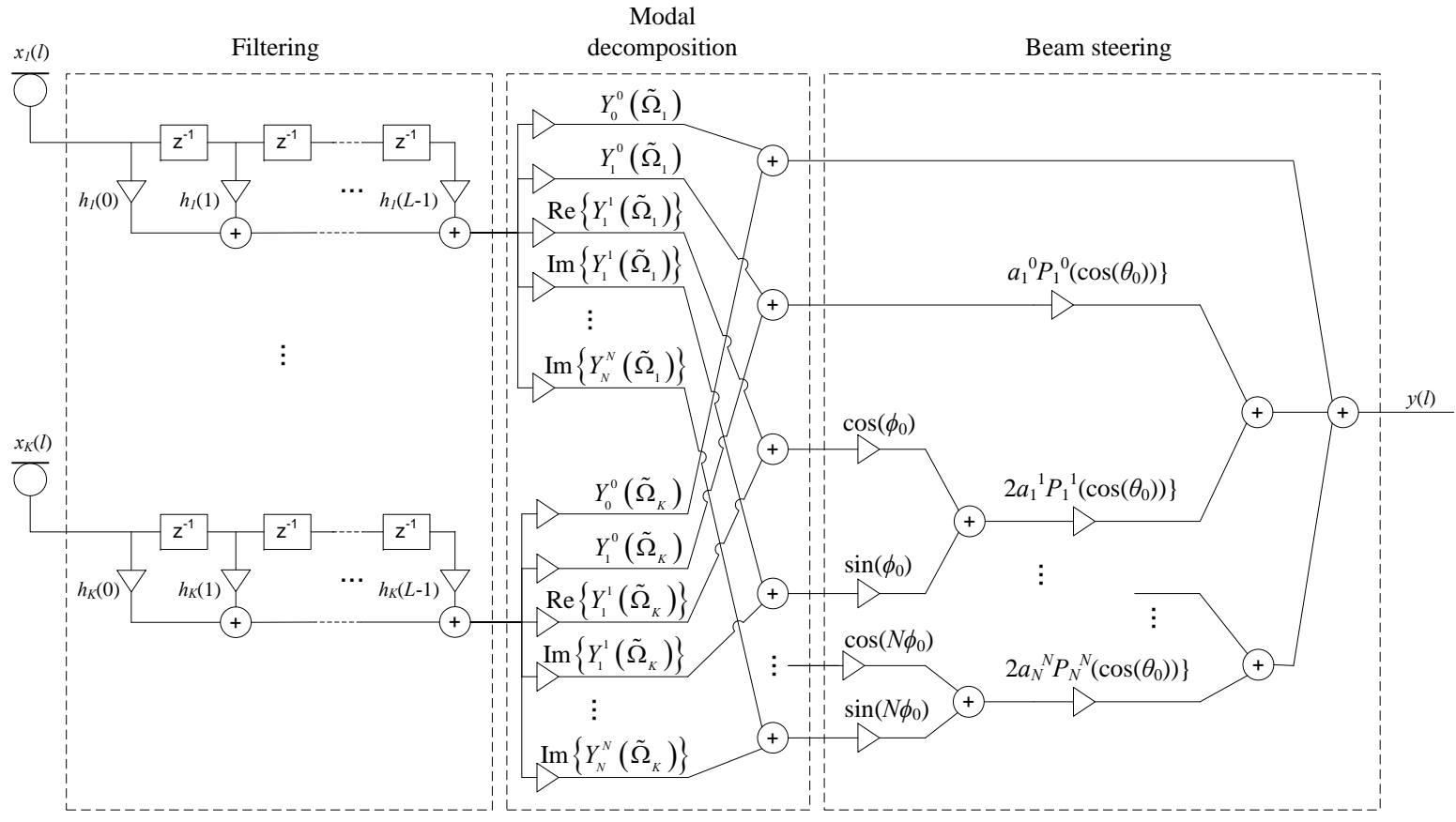

Figure 4. Proposed time domain beamformer structure.

where FIR filters are used to approximate the frequency dependent weights in the frequency domain design. Hence, as long as the length of the FIR filters is sufficiently long to provide a close approximation of the complex weights, the minimum number of required sensors is also $(N+1)^{2}$.

\section{Steerability of the main-beam}

Suppose the design expression given by (15) for frequency domain, or (35) for time domain, is satisfied completely. Then, both the beampattern expressions (14) and (34) will equal exactly the desired beampattern (10). Since (10) is identical to the beampattern expressions in [2], [6], this means the proposed beamformer design can be steered in the same way as the existing spherical beamformer designs, i.e. by modulating with $Y_{n}^{m}\left(\Omega_{0}\right)$, without affecting its beampattern.

\section{Effect of array rotation}

In the proposed design, the beamformer weights depend on the position of the sensors. Accordingly, the orientation of the sensor array will affect its beampattern, which is not the case with the existing spherical beamformers. In order to analyse this effect, an array rotation of $\Omega_{r}=\left(\theta_{r}, \phi_{r}\right)$ is introduced into (9), which yields

$$
\begin{aligned}
B\left(f, \Omega, \Omega_{0}\right) & =\sum_{n=0}^{\infty} \sum_{m=-n}^{n}\left[Y_{n}^{m}(\Omega)\right]^{*} Y_{n}^{m}\left(\Omega_{0}\right) b_{n}(k a) \\
& \times \oint_{\tilde{\Omega} \in S^{2}} w^{*}(f, \tilde{\Omega}) Y_{n}^{m}\left(\tilde{\Omega}+\Omega_{r}\right) d \tilde{\Omega} .
\end{aligned}
$$

Note that to avoid any approximation error in the analysis, due to the discretisation of sensors and temporal sampling, (9) is used instead of (14) or (34) in (47). Note also that the term $\Omega_{r}$ is not introduced in the weights $w^{*}(f, \tilde{\Omega})$ since the weights are fixed after the design. Substituting (2) into (47), it can be shown that

$$
\begin{aligned}
B\left(f, \Omega, \Omega_{0}\right) & =\sum_{n=0}^{\infty} \sum_{m=-n}^{n}\left[Y_{n}^{m}(\Omega)\right]^{*} Y_{n}^{m}\left(\theta_{0}, \phi_{0}+\phi_{r}\right) b_{n}(k a) \\
& \times \oint_{\tilde{\Omega} \in S^{2}} w^{*}(f, \tilde{\Omega}) Y_{n}^{m}\left(\tilde{\theta}+\theta_{r}, \tilde{\phi}\right) d \tilde{\Omega}
\end{aligned}
$$

For $\theta_{r}=0,(48)$ shows that a mechanical rotation of the array by $\phi_{r}$ in the azimuth plane results merely in a rotation of the beampattern in that plane by the same angle (c.f. (9)).

Unfortunately, a similar property does not hold for rotation in elevation, since $\theta_{r}$ is embedded in the argument of the associated Legendre polynomials (see (2)). Using (13), the integral term in (48) becomes

$$
\begin{aligned}
\oint_{\tilde{\Omega} \in S^{2}} w^{*} & (f, \tilde{\Omega}) Y_{n}^{m}\left(\tilde{\theta}+\theta_{r}, \tilde{\phi}\right) d \tilde{\Omega}=\sum_{n=0}^{\infty} \sum_{m=-n}^{n} \frac{\alpha_{n}^{m}(f)}{b_{n}(k a)} \\
& \times \oint_{\tilde{\Omega} \in S^{2}}\left[Y_{n}^{m}(\tilde{\Omega})\right]^{*} Y_{n}^{m}\left(\tilde{\theta}+\theta_{r}, \tilde{\phi}\right) d \tilde{\Omega} .
\end{aligned}
$$

Since with rotation, $\left[Y_{n}^{m}(\tilde{\Omega})\right]^{*}$ and $Y_{n}^{m}\left(\tilde{\theta}+\theta_{r}, \tilde{\phi}\right)$ in (49) are no longer orthogonal, this means that rotation in elevation will distort the beampattern. However, in most applications, this effect is minor since for small values of $\theta_{r}$, the distortion is negligible as illustrated in Section V-C.

\section{E. Computational complexity}

In order to compare the computational complexity of the proposed spherical beamformer to the existing spherical beamformers, the computational complexity is assessed in terms of number of real multiplications (RM) and real additions (RA). It is assumed that a single complex multiplication is 
Table I

COMPARISON OF COMPUTATIONAL COMPLEXITY FOR FREQUENCY DOMAIN DESIGN.

\begin{tabular}{|l|l|c|c|}
\hline \multicolumn{2}{|c|}{ System block } & Existing & Proposed \\
\hline \multirow{3}{*}{ Modal decomposition } & RM & $4 K(N+1)^{2}$ & $4 K(N+1)^{2}$ \\
\cline { 2 - 4 } & RA & $2(2 K-1)(N+1)^{2}$ & $2(2 K-1)(N+1)^{2}$ \\
\cline { 2 - 4 } Beam steering & RM & $4(N+1)^{2}$ & $4(N+1)^{2}$ \\
\cline { 2 - 4 } & RA & $2(2 N+1)(N+1)$ & $4(N+1)^{2}-2$ \\
\cline { 2 - 4 } Filtering & RM & $4(N+1)$ & $4 K$ \\
\cline { 2 - 4 } Total & RA & $2(2 N+1)$ & $2 K$ \\
\cline { 2 - 4 } & RM & $4\left((K+1)(N+1)^{2}+(N+1)\right)$ & $4\left((K+1)(N+1)^{2}+K\right)$ \\
\cline { 2 - 4 } & RA & $2\left((2 K+1)(N+1)^{2}+N\right)$ & $2\left((2 K+1)(N+1)^{2}+K-1\right)$ \\
\hline
\end{tabular}

equivalent to $4 \mathrm{RM}$ and $2 \mathrm{RA}$ while a complex addition is equivalent to $2 \mathrm{RA}$. In addition, it is assumed that all weights, such as the beamformer weights and the spherical harmonic coefficients for modal decomposition and beam steering have been precomputed and stored in memory. Table I compares the required number of RM and RA for the proposed spherical beamformer design in the frequency domain against the existing design of [2]. The required number of RM and RA is per frequency bin. Table II compares the computational complexity of the proposed design in time domain against the existing design of [6], where the required number of RM and $\mathrm{RA}$ is for the whole spectral range of interest.

For a given $K, N$ and $L$ with $N<K$, it seems the proposed method will result in a higher computational load. However, if the orthonormality criterion is taken into account, i.e. for the existing designs, a minimum of $K=1.5(N+1)^{2}$ sensors are required [14], whereas for the proposed design, a minimum of $K=(N+1)^{2}$ sensors are required, then a different conclusion can be drawn. For the frequency domain design, the proposed design has a lower computational load compared to the existing designs in all cases as shown in Fig. 5. For the time domain designs, the proposed design achieves lower computational load if

$$
L<\frac{(N+1)^{3}}{2 N}
$$

where (50) is obtained by equating the corresponding total $\mathrm{RM}$ and RA counts from Table II after substituting $K=$ $1.5(N+1)^{2}$ for the existing method and $K=(N+1)^{2}$ for the proposed method. For example, to design a beamformer with spherical harmonic order of $N=5$, the proposed design has a computational advantage if the filter length is chosen to be $L<21.6$. This dependency on the filter length $L$ is shown in Fig. 6, where the dashed lines, plotted for $L \in[4,5152]$, represent the locations where the number of operations for both the proposed and the existing designs coincide for different values of $L$. The region below the dashed is where the proposed method has a computational advantage.

\section{Design EXAMPLES}

To illustrate the performance of the proposed design, a number of time domain design examples based on (40) are presented. The design parameters are specified in Table III, where the sensors are assumed to be omni-directional microphones mounted on a rigid sphere.
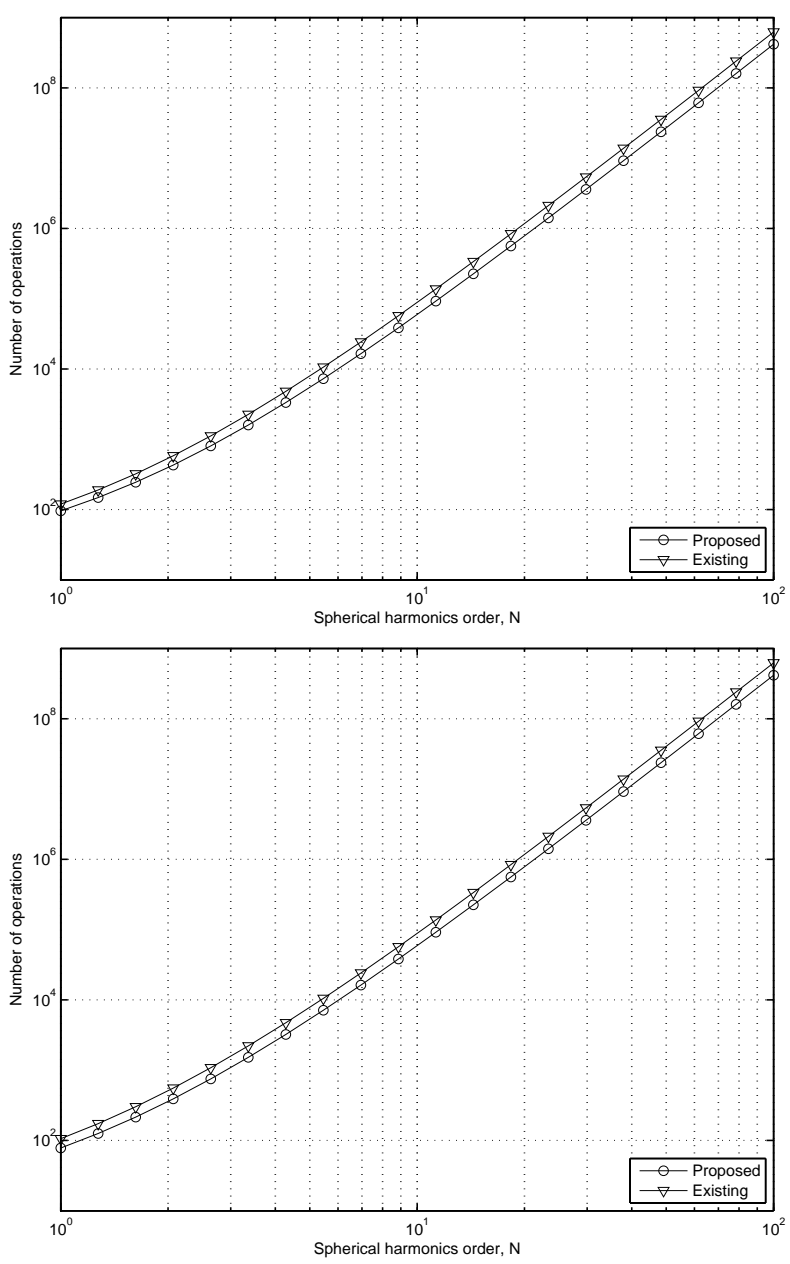

Figure 5. Comparison of required number of RM (top) and RA (bottom) for frequency domain designs.

Table III

DESIGN PARAMETERS.

\begin{tabular}{|l|c|}
\hline \multicolumn{1}{|c|}{ Parameters } & Value \\
\hline Highest spherical harmonics order, $N$ & 5 \\
\hline Number of sensor, $K$ & 36 \\
\hline FIR filter length, $L$ & 64 \\
\hline Sampling frequency, $f_{S}$ & $8000 \mathrm{~Hz}$ \\
\hline Spectral passband, $\Omega_{p b}$ & {$[200,3800] \mathrm{Hz}$} \\
\hline Radius of spherical array, $a$ & $4 \mathrm{~cm}$ \\
\hline
\end{tabular}


Table II

COMPARISON OF COMPUTATIONAL COMPLEXITY FOR TIME DOMAIN DESIGN.

\begin{tabular}{|c|c|c|c|}
\hline \multicolumn{2}{|c|}{ System block } & Existing & Proposed \\
\hline \multirow{3}{*}{ Modal decomposition } & RM & $K(N+1)^{2}$ & $K(N+1)^{2}$ \\
\cline { 2 - 4 } & RA & $(K-1)(N+1)^{2}$ & $(K-1)(N+1)^{2}$ \\
\cline { 2 - 4 } Beam steering & RM & $\left(3 N^{2}+5 N\right) / 2$ & $\left(3 N^{2}+5 N\right) / 2$ \\
\cline { 2 - 4 } & RA & $N(N+1)$ & $N(N+2)$ \\
\cline { 2 - 4 } Filtering & RM & $L(N+1)$ & $K L$ \\
\cline { 2 - 4 } & RA & $L(N+1)-1$ & $K(L-1)$ \\
\cline { 2 - 4 } Total & \multirow{2}{*}{ RM } & $(N+1)\left(K(N+1)+\frac{3}{2} N\right)$ & $(N+1)\left(K(N+1)+\frac{3}{2} N\right)$ \\
& & $+N+L(N+1)$ & $+N+K L$ \\
\cline { 2 - 4 } & RA & $K N(N+2)-1$ & $K N(N+2)-1$ \\
& & $+(N+1)(L-1)+K$ & $+K L$ \\
\hline
\end{tabular}
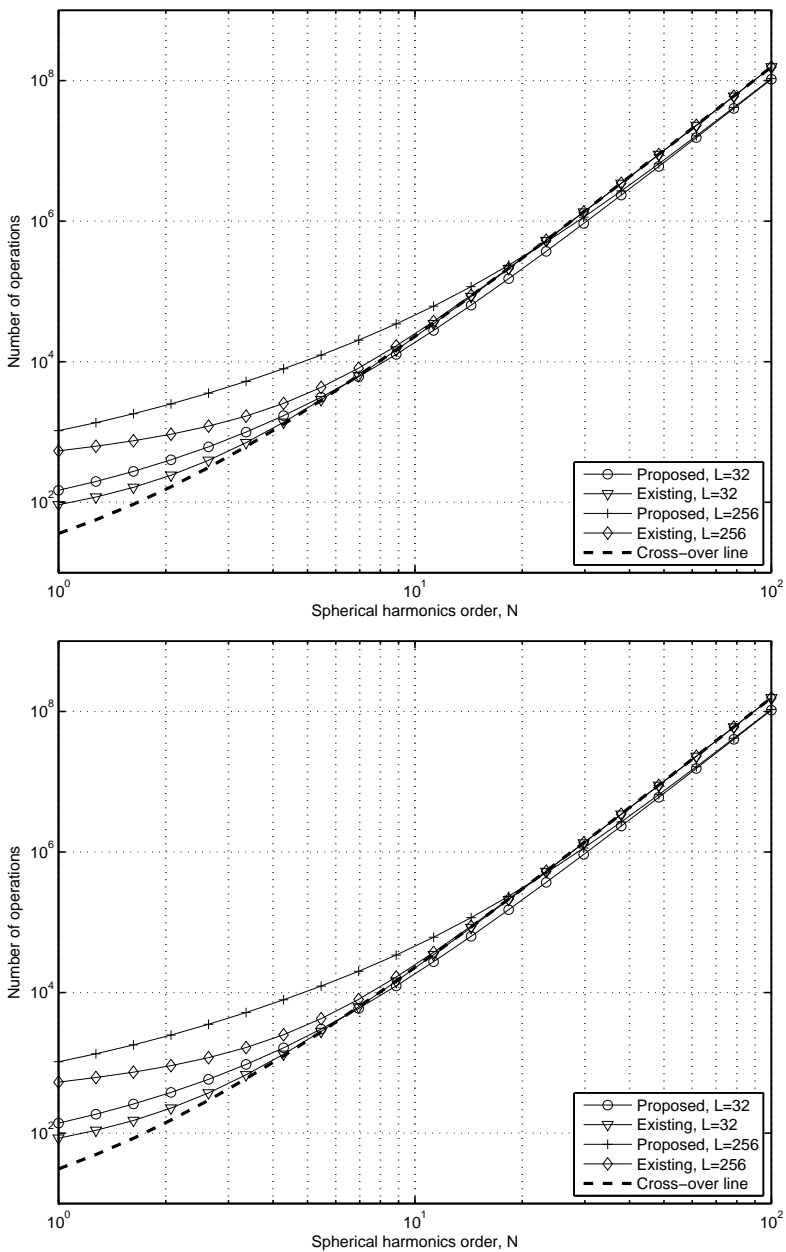

Figure 6. Comparison of required number of RM (top) and RA (bottom) for time domain designs.

With regards to the sensor configurations, a quasi-uniform configuration [12] shown in Fig. 7 and a random configuration shown in Fig. 8 are considered. The sensor positions for the random configuration are given by

$$
\tilde{\theta}_{s}=\cos ^{-1}(2 u-1), \quad \tilde{\phi}_{s}=2 \pi v
$$

where $u, v \sim \mathcal{U}(0,1)$, in order to have sensors uniformly distributed over the surface of the sphere [24].

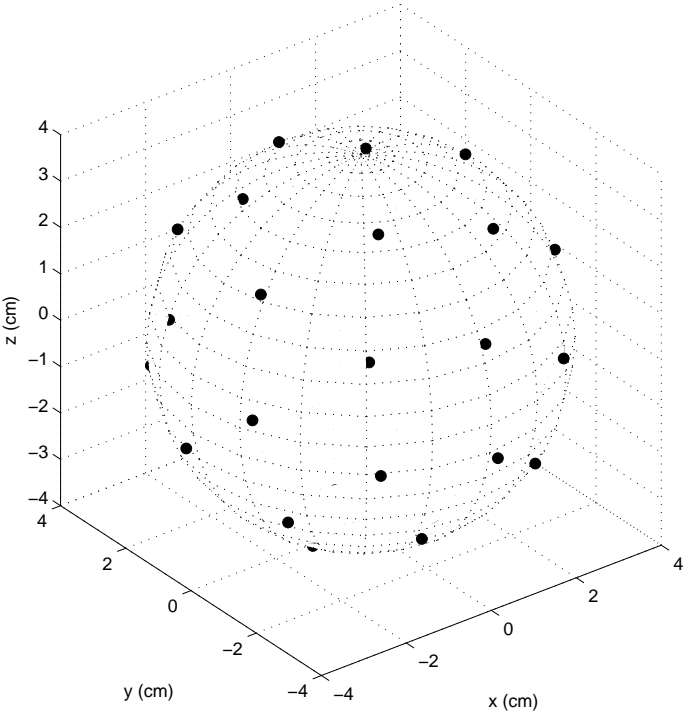

Figure 7. Uniform sensor configuration.

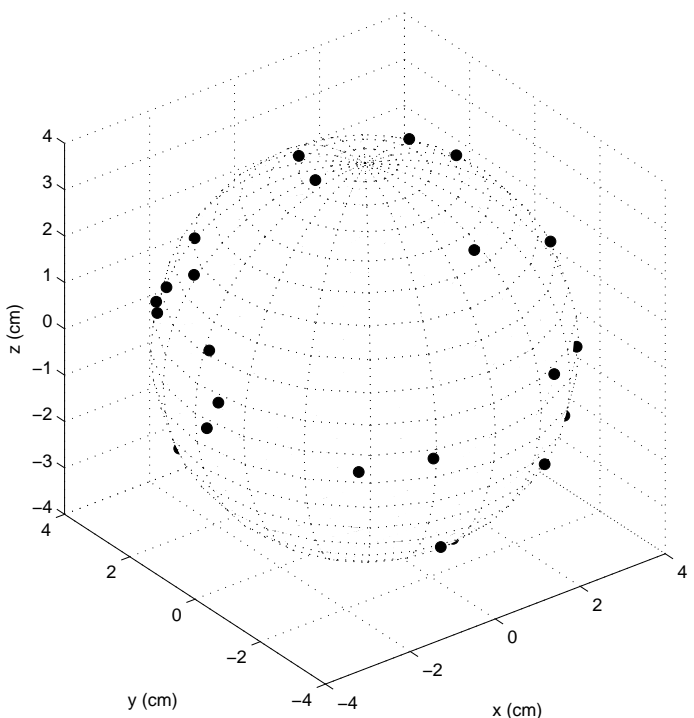

Figure 8. Random sensor configuration. 


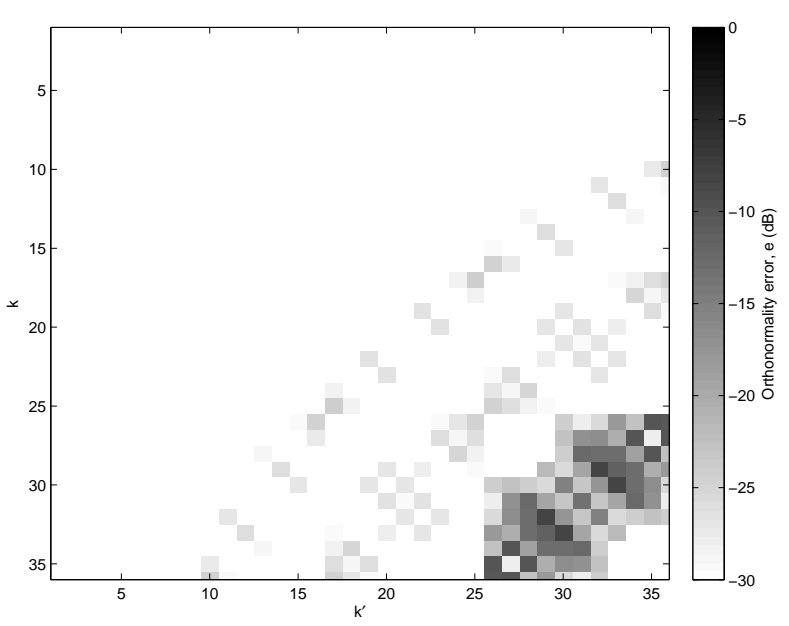

Figure 9. Plot of the orthonormality error (52) for the uniform sensor configuration.

\section{A. Sensor configurations and orthonormality criterion}

In this section, the limitation imposed by the orthonormality criterion (6) on the existing methods is illustrated. For the uniform sensor configuration shown in Fig. 7, the absolute error squared of the orthonormality criterion, defined by

$$
\begin{aligned}
& e\left(n, m, n^{\prime}, m^{\prime}\right) \\
& \quad=\left|\sum_{s} \alpha_{s}\left[Y_{n}^{m}\left(\tilde{\Omega}_{s}\right)\right]^{*} Y_{n^{\prime}}^{m^{\prime}}\left(\tilde{\Omega}_{s}\right)-\delta_{m-m^{\prime}} \delta_{n-n^{\prime}}\right|^{2},
\end{aligned}
$$

where the index $k$ is defined by $k=n(n+1)+m+1$, and $k^{\prime}$ is defined similarly, is shown in Fig. 9. The white patch on the upper left corner of Fig. 9 shows that the uniform configuration satisfies (6) up to around $k \leq 21 \cap k^{\prime} \leq 21$ (which corresponds to $N=3$, rounded down). For $k$ and $k^{\prime}>$ 21 , the violation of the orthonormality criterion increases as indicated by the appearances of dark patches (indicating large error) in the lower right corner. This limits the operable order of the existing beamfomer to $N=3$ (where the low frequency components start to increase), as shown by the beampatterns in Fig. 10, evaluated for $N=2$ and $N=3$ using the existing method of [6]. This limiting factor was discussed by Hardin and Sloane [14].

In the case of the proposed method (see Section IV-B), given the same number of sensors $K=36$, it can resolve more spherical harmonics (up to $N=5$ ) without any spatial aliasing. Fig. 11 shows the beampattern of the proposed method. Moreover, for the random configuration shown in Fig. 8, which does not satisfy orthonormality criterions at all (indicated by large orthonormality error for all $k$ and $k^{\prime}$ in Fig. 12), the proposed method can still be used to design a modal beamformer up to $N=5$ order of spherical harmonics. The beampattern of this design is shown in Fig. 13.

\section{B. Steerability performance}

In this section, the ability of the proposed method to employ any arbitrary sensor configuration without sacrificing its steerability is illustrated and compared against the design method of
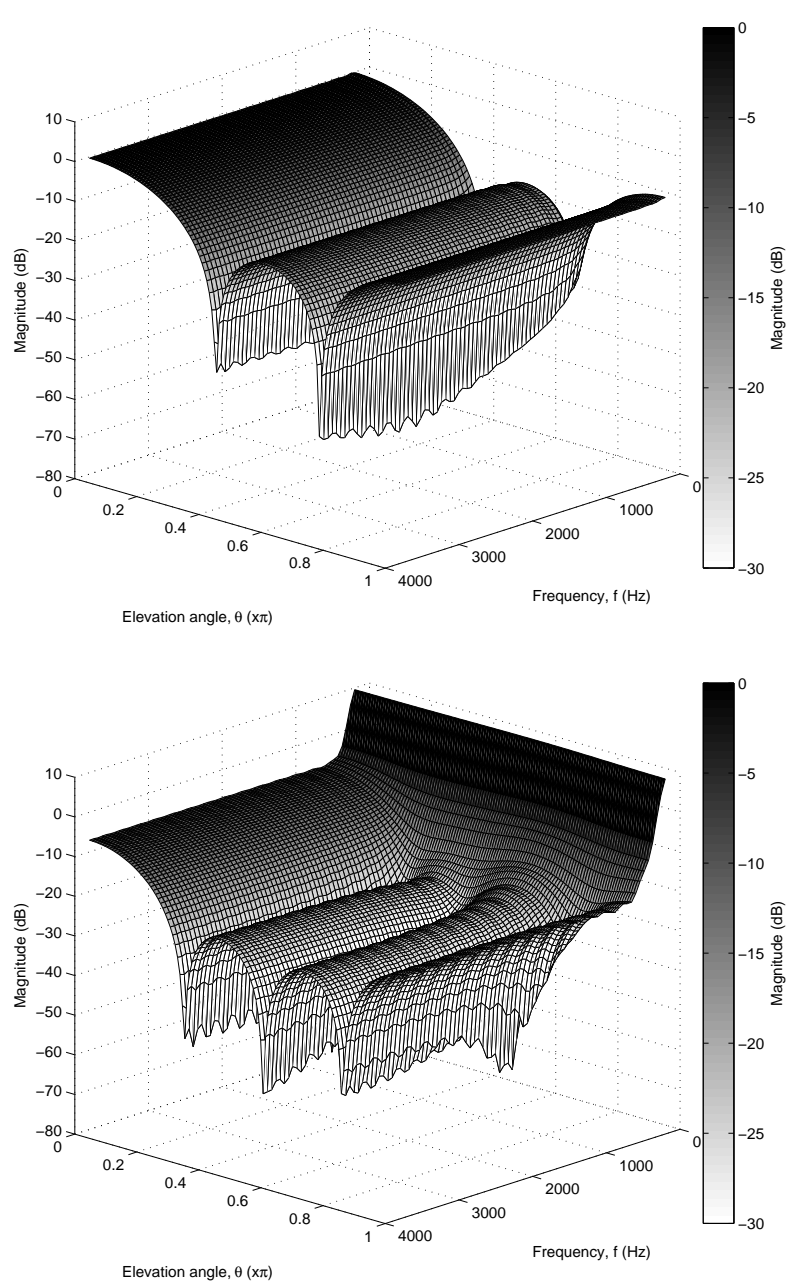

Figure 10. Beampattern for the existing design (uniform sensor configuration) steered to $\Omega_{0}=(0,0)$, evaluated for $N=2$ (top) and $N=3$ (bottom).

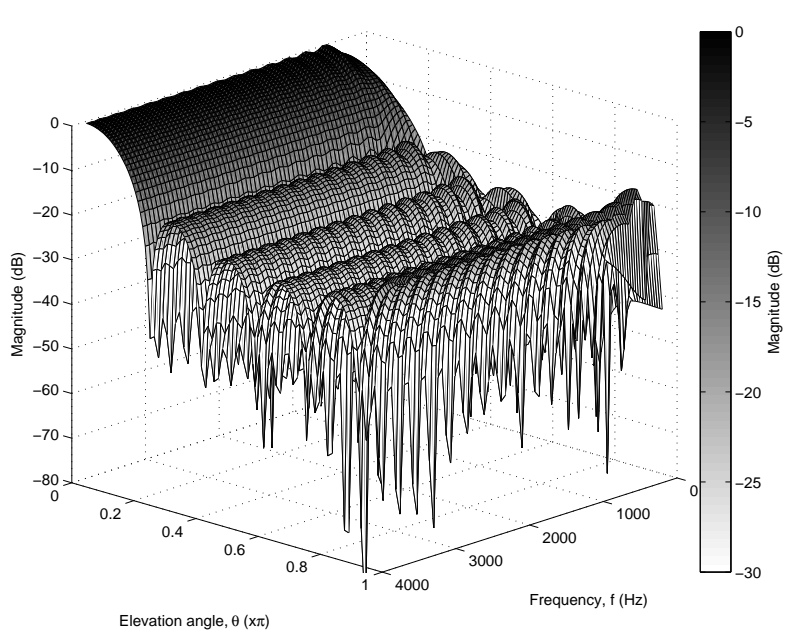

Figure 11. Beampattern for the proposed design (uniform sensor configuration) steered to $\Omega_{0}=(0,0)$ and evaluated for $N=5$. 


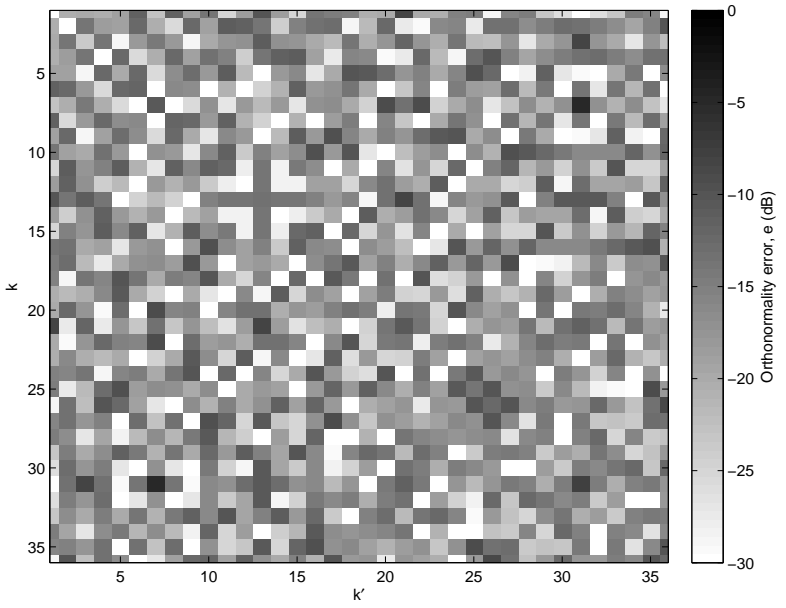

Figure 12. Plot of the orthonormality error (52) for the random sensor configuration.

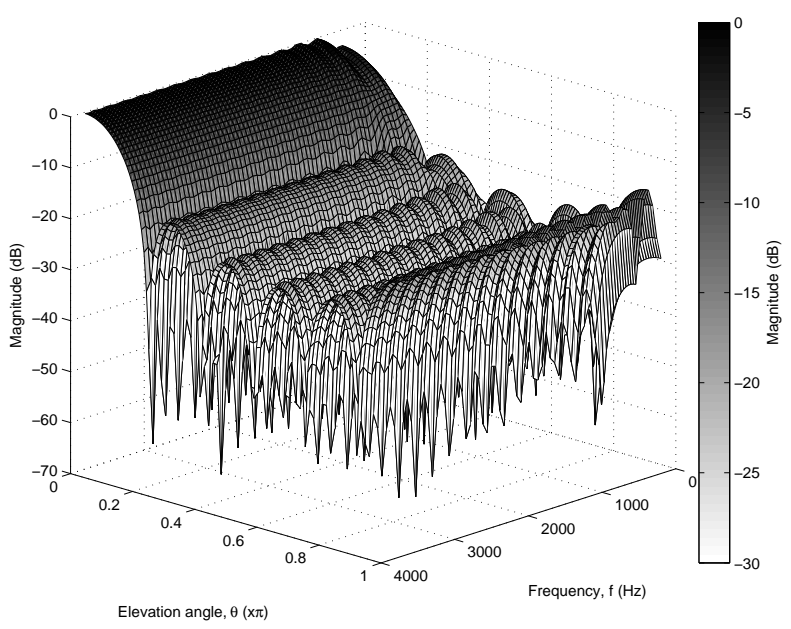

Figure 13. Beampattern for the proposed design (random sensor configuration) steered to $\Omega_{0}=(0,0)$ and evaluated for $N=5$.

[6]. For the proposed method, the sensor configurations shown in Figs. 7 and 8 are considered. For the existing method, it is assumed that (6) holds completely up to $N=5$, either using equiangle or Gaussian sampling scheme (see Section II-B).

The performance of the designs are evaluated in terms of the integral of directivity covering the whole range $\Omega_{0} \in$ $\left\{\theta_{0}, \phi_{0}: 0 \leq \theta_{0} \leq \pi, 0 \leq \phi_{0} \leq 2 \pi\right\}$

$$
D(f)=\oint_{\Omega_{0}} D\left(f, \Omega_{0}\right) d \Omega_{0}
$$

where directivity $D\left(f, \Omega_{0}\right)$ is defined as [25]

$$
D\left(f, \Omega_{0}\right)=\frac{4 \pi\left|B\left(f, \Omega_{0}, \Omega_{0}\right)\right|^{2}}{\int_{\Omega}\left|B\left(f, \Omega, \Omega_{0}\right)\right|^{2} d \Omega} .
$$

Figs. 14 and 15 show the integral of directivity of the nonrobust and robust (with $\rho=2$ ) designs, respectively. These figures show that the proposed designs, though using sensor configurations that do not satisfy (6) (see Figs. 9 and 12), still have performance similar to the existing design (except

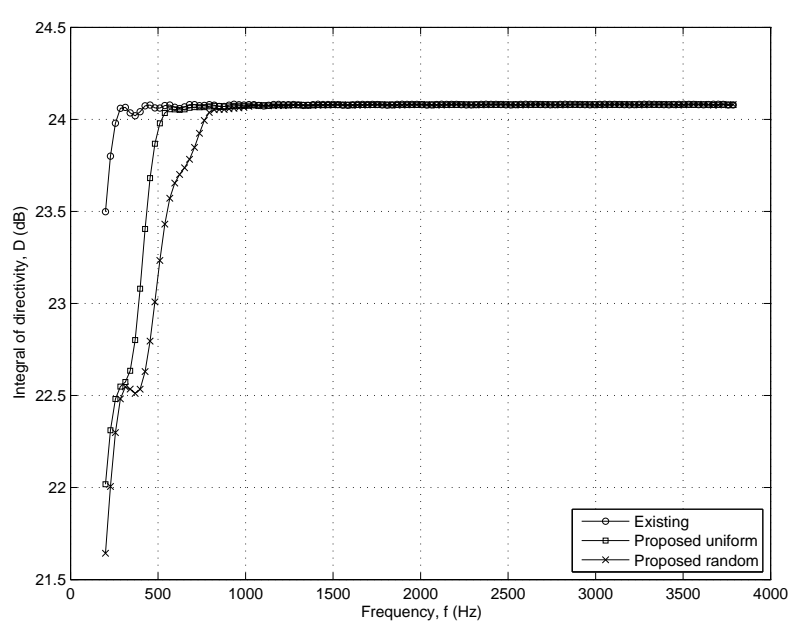

Figure 14. Integral directivity for non-robust designs.

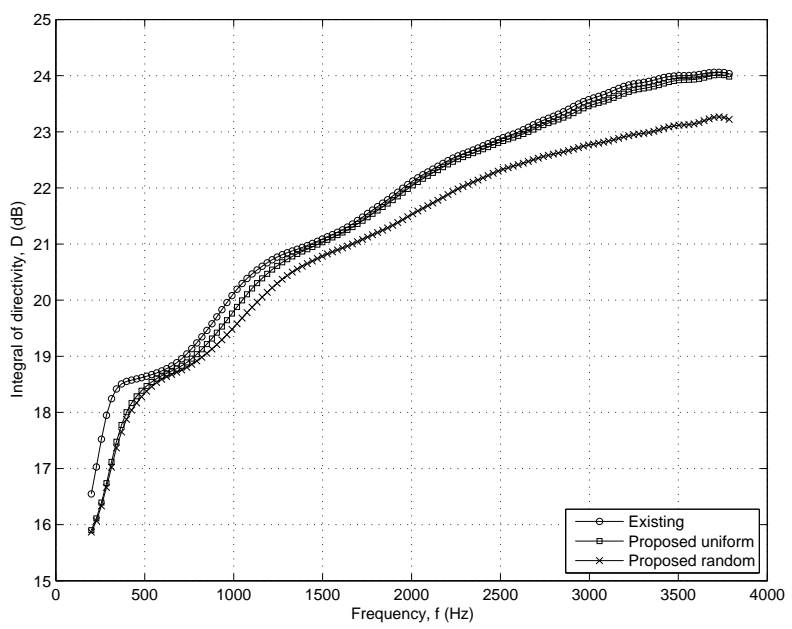

Figure 15. Integral directivity for robust designs (with $\rho=2$ ).

for the slight degradation at low frequencies). This means the proposed designs are not limited by the orthonormality criterion (6), and moreover, are able to retain the desirable property of steerability.

The WNG for the non-robust and robust designs are shown in Fig. 16. Low WNG indicates that the beamformers are not robust, and this happens at the low frequency of the nonrobust designs. For the robust designs, their WNG measures are high and constant indicating their robustness (especially at low frequency).

\section{Performance with rotated array}

In order to illustrate the discussion in Section IV-D, the beampattern of the proposed beamformer with random sensor configuration, steered to $\Omega_{0}=(3 \pi / 4, \pi / 3)$ is shown in Fig. 17. The beampattern with the same beamformer, but with its sensor array rotated by $\Omega_{r}=(0, \pi / 3)$ along the azimuth plane, is shown in Fig. 18. The main-beam, under steering and array rotation, is now located at $\Omega_{0}+\Omega_{r}$ (compare Fig. 17 with 18).

In the case of array rotation in elevation, Fig. 19 shows the directivity (54) of the beamformer with an array rotation 


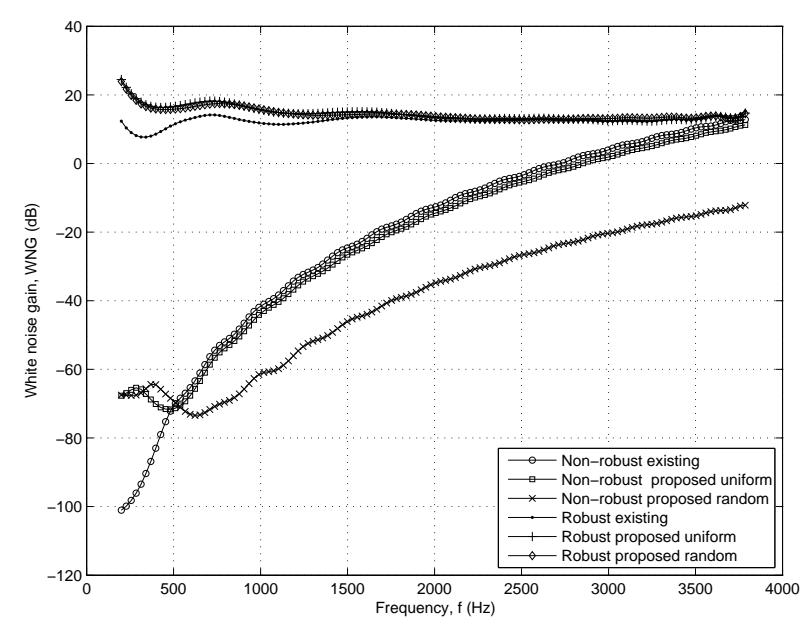

Figure 16. WNG measures for non-robust and robust (with $\rho=2$ ) designs.

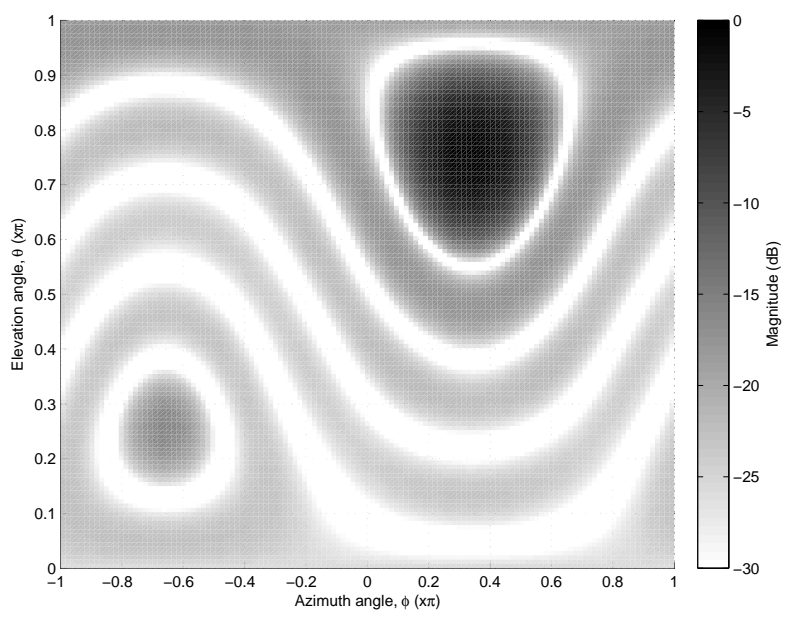

Figure 17. Beampattern of proposed design (random sensor configuration) steered to $\Omega_{0}=(3 \pi / 4, \pi / 3)$.

of $\theta_{r} \in[0, \pi]$ and $\phi_{r}=0$. Although the array rotation in elevation will decrease the beamformer's directivity, the effect is minor for small $\theta_{r}$. For example, for a $1 \mathrm{~dB}$ drop in directivity, the range of $\theta_{r}$ is around $0 \leq \theta_{r} \leq 0.3 \pi$.

\section{CONCLUSION}

A design method based on spherical harmonics for filterand-sum beamformers with straightforward steering capability has been proposed. The proposed method allows for flexibility in sensor configuration and straightforward beam steering with a beampattern that is invariant to the steering direction. Flexibility in the sensor configuration to allow arbitrary configurations is achieved through the filter-and-sum beamformer while the straightforward steering property of spherical harmonic beamforming is achieved through spherical harmonic decomposition and modulation. In addition, it is shown that the proposed method requires fewer sensors to achieve similar performance as the existing spherical harmonic beamformers for the same order of spherical harmonics. The main trade-off of the proposed method, as compared to the existing beamformers, is that prior knowledge of the sensor

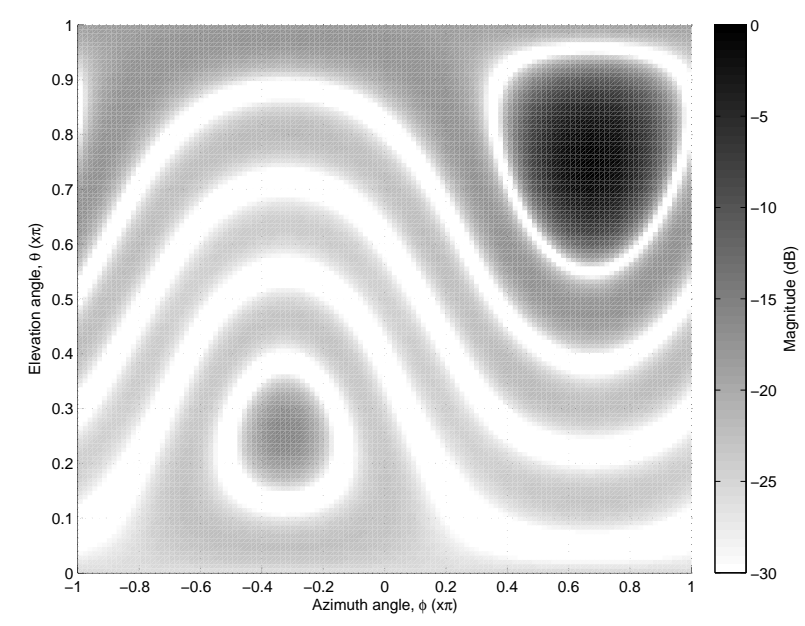

Figure 18. Beampattern of proposed design (random sensor configuration) steered to $\Omega_{0}=(3 \pi / 4, \pi / 3)$ and with array rotation of $\Omega_{r}=(0, \pi / 3)$.

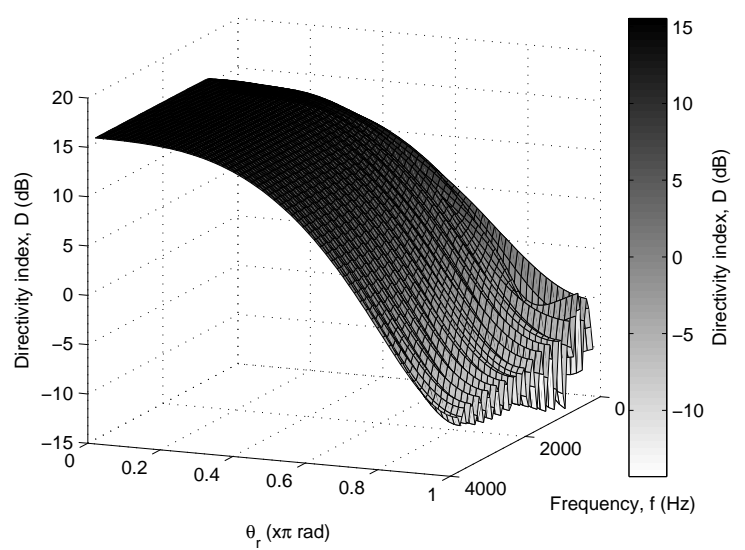

Figure 19. DI of proposed design (random sensor configuration) with array rotation of $\theta_{r} \in[0, \pi]$ and $\phi_{r}=0$.

positions are required, which results in its beampattern being dependent on the array orientation. However, the effect of such dependency is minor since small changes in the array orientation does not cause significant distortion to the beampattern. Robustness of the proposed designs can be achieved by constraining its WNG.

\section{REFERENCES}

[1] D. Ward, Z. Ding, and R. Kennedy, "Broadband doa estimation using frequency invariant beamforming," IEEE Trans. Signal Process., vol. 46, no. 5, pp. $1463-1469$, may 1998.

[2] J. Meyer and G. Elko, "A highly scalable spherical microphone array based on an orthonormal decomposition of the soundfield," in Proc. IEEE International Conference Acoustics, Speech, and Signal Processing, vol. 2, Orlando, FL, USA, 13-17 May 2002, pp. 1781-1784.

[3] T. D. Abhayapala and D. B. Ward, "Theory and design of high order sound field microphones using spherical microphone array," in Proc. IEEE International Conference Acoustics, Speech, and Signal Processing, vol. 2, Orlando, FL, USA, 13-17 May 2002, pp. 1949-1952.

[4] B. K. Lau and Y. H. Leung, "A dolph-chebyshev approach to the synthesis of array patterns for uniform circular arrays," in Circuits and Systems, 2000. Proceedings. ISCAS 2000 Geneva. The 2000 IEEE International Symposium on, vol. 1, 2000, pp. 124-127 vol.1. 
[5] S. Yan, H. Sun, U. Svensson, X. Ma, and J. Hovem, "Optimal modal beamforming for spherical microphone arrays," IEEE Trans. Audio, Speech, Language Process., vol. 19, no. 2, pp. 361-371, Feb 2011.

[6] S. Yan, H. Sun, X. Ma, U. Svensson, and C. Hou, "Time-domain implementation of broadband beamformer in spherical harmonics domain," IEEE Trans. Audio, Speech, Language Process., vol. 19, no. 5, pp. 1221 -1230 , Jul 2011.

[7] E. Fisher and B. Rafaely, "Near-field spherical microphone array processing with radial filtering," IEEE Trans. Audio, Speech, Language Process., vol. 19, no. 2, pp. 256 -265, Feb 2011.

[8] B. Rafaely, B. Weiss, and E. Bachmat, "Spatial aliasing in spherical microphone arrays," IEEE Trans. Signal Process., vol. 55, no. 3, pp. 1003 -1010, Mar 2007.

[9] J. R. Driscoll and D. M. Healy, "Computing fourier transforms and convolutions on the 2-sphere," Advances in Applied Mathematics, vol. 15, no. 2, pp. $202-250,1994$.

[10] M. J. Mohlenkamp, "A fast transform for spherical harmonics," Journal of Fourier Analysis and Applications, vol. 5, pp. 159-184, 1999.

[11] G. Arfken and H. Weber, Mathematical Methods for Physicists, 5th ed. San Diego, Academic Press, 2001.

[12] J. Fliege and U. Maier, "The distribution of points on the sphere and corresponding cubature formulae," IMA Journal of Numerical Analysis, vol. 19, no. 2, pp. 317-334, 1999.

[13] A. Katanforoush and M. Shahshahani, "Distributing points on the sphere, i," Experimental Mathematics, vol. 12, no. 2, pp. 199-209, 2000.

[14] R. H. Hardin and N. J. A. Sloane, "Mclaren's improved snub cube and other new spherical designs in three dimensions," ArXiv Mathematics, Jul 2002.

[15] Z. Li and R. Duraiswami, "Flexible and optimal design of spherical microphone arrays for beamforming," IEEE Trans. Audio, Speech, Language Process., vol. 15, no. 2, pp. 702 -714, Feb 2007.

[16] M. Kajala and M. Hamalainen, "Filter-and-sum beamformer with adjustable filter characteristics," in Proc. IEEE International Conference Acoustics, Speech, and Signal Processing, vol. 5, Salt Lake City, UT, 7-11 May 2001, pp. 2917-2920.

[17] L. C. Parra, "Steerable frequency-invariant beamforming for arbitrary arrays," J. Acoust. Soc. Am., vol. 119, no. 6, pp. 3839-3847, 2006.

[18] C. C. Lai, S. Nordholm, and Y. H. Leung, "Design of robust steerable broadband beamformers incorporating microphone gain and phase error characteristics," in Proc. IEEE International Conference Acoustics, Speech, and Signal Processing, Prague, 22-27 May 2011.

[19] D. H. Johnson and D. E. Dudgeon, Array Signal Processing - Concepts and techniques. Prentice Hall, 1993.

[20] E. G. Williams, Fourier Acoustics: Sound Radiation and Nearfield Acoustical Holography. Academic Press, New York, 1999.

[21] B. Rafaely, "Analysis and design of spherical microphone arrays," IEEE Trans. Speech, Audio Process., vol. 13, no. 1, pp. 135-143, Jan 2005.

[22] E. N. Gilbert and S. P. Morgan, "Optimum design of directive antenna arrays subject to random deviations," Bell Syst. Tech. J., vol. 34, pp. 637-663, May 1955.

[23] J. F. Sturm, "Using sedumi 1.02, a matlab toolbox for optimization over symmetric cones," Optimization Methods and Software, vol. 11-12, pp. 625-653, 1999.

[24] W. Feller, An Introduction to Probability Theory and Its Applications, Vol. 2, 3rd ed. John Wiley \& Sons, Inc, New York, 1971.

[25] J. Bitzer and K. U. Simmer, "Superdirective microphone arrays," in Microphone Arrays, M. Brandstein and D. Ward, Eds. Springer-Verlag Berlin Heidelberg, New York, 2001, ch. 2, pp. 19-38.

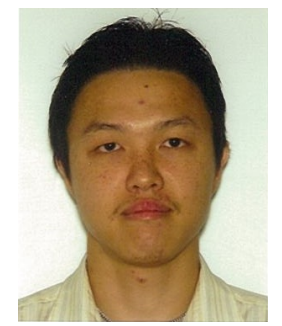

Chiong Ching Lai received his B. E. (Hons) in Electronics and Communications Engineering from Curtin University, Perth, Australia in 2007. He is currently pursuing Ph.D. degree in the Department of Electrical and Computing Engineering of Curtin University, Australia. Since Dec 2010, he has been a Research Assistant for the Communication Signal Processing research group in his department. His research interests include steerable broadband beamforming, source localisation and speech enhancement.

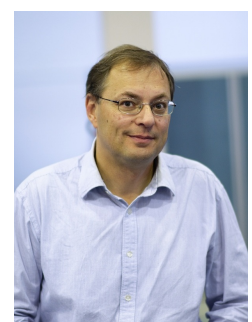

Sven Nordholm (Professor, Signal Processing, Curtin University of Technology). Dr. S. Nordholm received his Ph.D. in Signal Processing in 1992, Licentiate of engineering 1989 and MscEE (Civilingenjör) 1983 all from Lund University, Sweden. He was one of the founding members of the Department of Signal Processing, Blekinge Institute of Technology, BTH, Sweden in 1990. At BTH he held positions as Lecturer, Senior Lecturer, Associate Professor and Professor. Since 1999 he has been at Curtin University of Technology in Perth, Western Australia. From 1999-2002 he was director of ATRI and Professor at Curtin University of Technology. From 2002-2009 he was director Signal Processing Laboratory, WATRI, Western Australian Telecommunication Research Institute, a joint institute between The University of Western Australia and Curtin University. Currently, he is Prof. Signal Processing and Head of Department, Dept. of Electrical and Computer Engineering, Curtin University. He is also Chief Scientist and co-founder of a start-up company Sensear providing voice communication in extreme noise conditions. He is an associate editor Eurasip Journal on Advances in Signal Processing. He is a Senior Member of IEEE and a member of IEEE TC AASP. His main research efforts have been spent in the fields of Speech Enhancement, Adaptive and Optimum Microphone Arrays, Acoustic Echo Cancellation, Adaptive Signal Processing, Sub-band Adaptive Filtering and Filter Design.

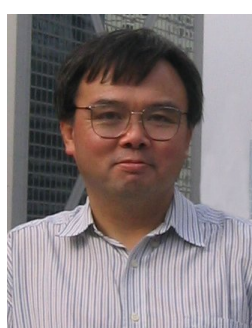

Yee Hong Leung (M'80) was born in Singapore in 1955. He obtained the B.E. (Hons) and Ph.D. degrees, both in electrical engineering, from the University of Western Australia, Australia, in 1977 and 1986, respectively. He was with the School of Electrical, Electronic and Computer Engineering, the University of Western Australia, as a Tutor from 1982 to 1986 and as a Lecturer from 1988 to 1994. From 1986 to 1988, he was with the Defence Science and Technology Organisation, South Australia as a Research Scientist. In 1994, he joined the Western Australian Telecommunications Research Institute, Curtin University, Australia, as a Senior Research Fellow. He was also associated with the Australian Telecommunications Cooperative Research Centre. He joined the Department of Electrical and Computer Engineering, Curtin University, in 2004 as a Senior Lecturer. His research interests are in adaptive and optimum signal processing, and their applications in antenna arrays and telecommunication systems. 